\title{
A Case of Cortical Tremor as a Variant of Cortical Reflex Myoclonus
}

\begin{tabular}{|l|l|l|}
\hline E. & Oguni \\
\hline A. & Hayashi \\
\hline A. & Ishii \\
\hline H. & Mizusawa \\
\hline S. & Shoji \\
\hline
\end{tabular}

Department of Neurology, Institute of Clinical Medicine, University of Tsukuba, Japan

Akito Hayashi, MD, Department of Neurology, Institute of Clinical Medicine, University of Tsukuba, Tsukuba-city, Ibaraki-ken 305 (Japan)

Myoclonus sometimes represents rhythmical movement and cortical tremor has been defined as a rhythmical movement accompanied by cortical reflex myoclonus [1]. We examined a patient with cortical tremor who demonstrated rhythmical muscle discharges evoked by a peripheral nerve stimulation.

A 64-year-old man had a 9-year history of postural tremor involving the hands and fingers. The patient also had generalized seizure, but there were no other neurological findings including dementia. Oral administration of valproate only had the effect of controlling his epileptic attack but had no effect on his tremor; neither had propranolol. Family history involved tremor of the hands, generalized epilepsy or both. Laboratory tests including urinalysis, blood cell count, and serum electrolytes were normal. Thyroid function, lactate and pyruvate levels in serum as well as CSF were all normal. Cranial MRI was normal. Surface EMG of the postural tremor recorded on his forearm revealed rhythmical discharges, which had a reciprocity and in part a co-contraction between antagonists (fig. la). The frequency of mechanical movement, monitored with an accelerometer attached to the hand and estimated through a fast Fourier transformation, was around 7.4 Hz and ranged from 6.3 to $9.4 \mathrm{~Hz}$ (fig. lb). EEG showed

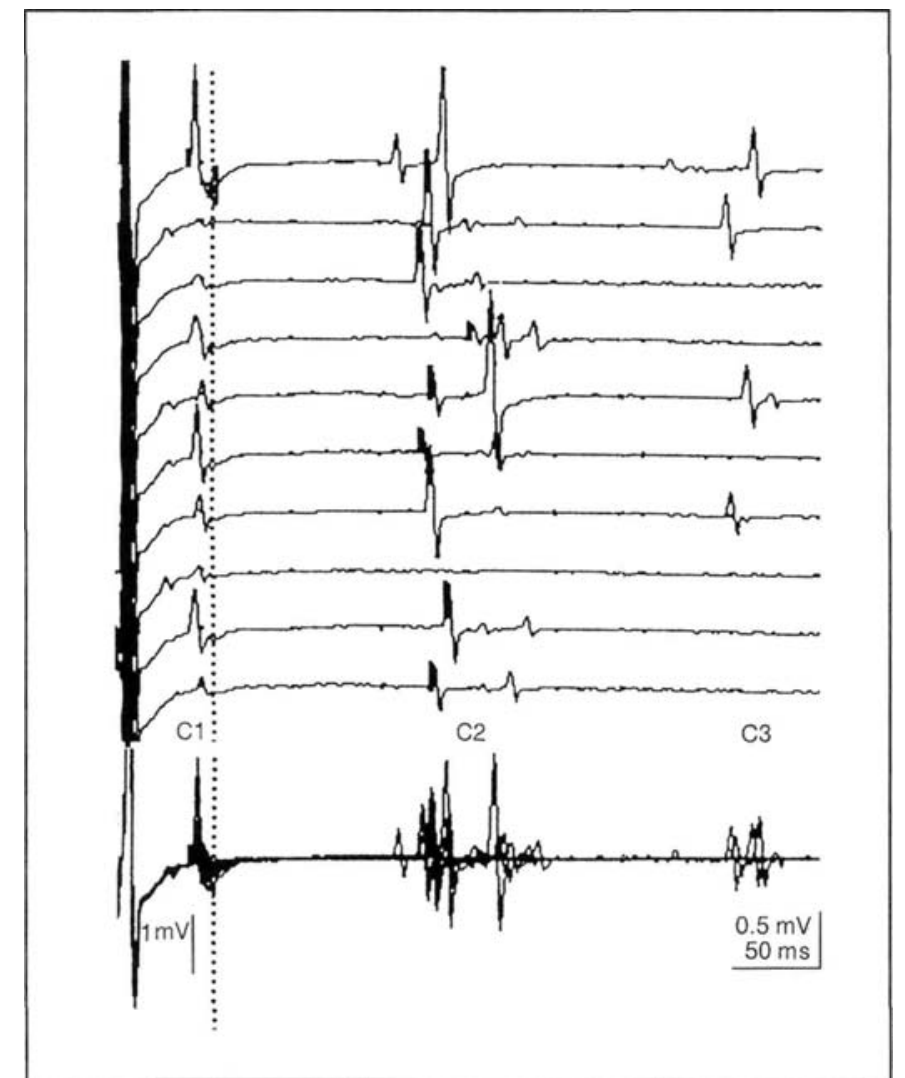

AVvAA/yVMA

$\underset{\text { flexor }}{\text { Wrist }} \frac{\text { FL. 44ijL.Li }}{i<n}$ 


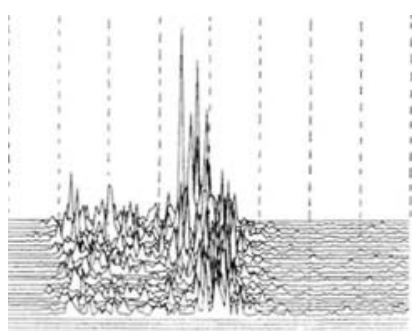

$16 \mathrm{~Hz}$

Fig. 1. a Surface EMG from wrist flexor (middle row) and wrist extensor (lower row), and movement monitor by accerelometer (upper row; ACC). These were reciprocal, and partially co-contracted muscle activity between antagonists, and the movement was sinusoidal, b Frequency spectrum of the sinusoidal movement; calculated by fast Fourier transformation. Each line shows the result of every 30-second sampling.

Fig. 2. Compound muscle action potentials from APB evoked by single electrical shock of median nerve. Each record shows raster mode and the bottom is displayed by superimposed mode. Reflex potentials demonstrate repetitive responses, CI is seen at $40 \mathrm{~ms}$ after M-wave, C2 and C3 are seen at around 200 and approximately $350 \mathrm{~ms}$, respectively.

\section{(1) 1995/S.Karger|AG, Basel}

\section{$\$ 8.00 / 0$}

sporadic spikes at the bilateral centroparietal region. SEPs elicited by a median nerve stimulation demonstrated marked increase of amplitude at P25 and N33 (48 uV), which satisfied the criteria of giant SEPs [25]. C-reflex from the thenar muscle was enhanced at a latency of $40 \mathrm{~ms}$. Jerk-locked averaging of monopolar EEG triggered by the tremor activity of thenar demonstrated premyoclonus cortical discharge timelocked to the muscle activities at a latency of approximately $16 \mathrm{~ms}$ to negative peak of cortical discharge from the onset of the muscle activities. Median nerve stimulation at rest elicited three clusters of repetitive responses in the thenar muscle on the superimposed record (fig. 2). The onset of the first response was well synchronized at the same latency of C-reflex. The latency of the second and third responses fluctuated between 156-324 and 344-478 ms, respectively. The second response frequently showed double discharges and the third was sometimes lacking. The intervals from the first response to the center of the second cluster (C1-C2) and from the center of the second to the third (C2-C3) was approximately $150 \mathrm{~ms}$. The frequency of these responses was about $7 \mathrm{~Hz}$, which was comparable to that of the tremor.

Cortical tremor is proposed as a variant of cortical reflex myoclonus by Ikeda et al. [1], with the characteristics of (1) essential tremor; (2) history of seizures or EEG abnormality in the family; (3) physiological findings consisting of cortical reflex myoclonus, and (4) no other neurological deficits such as dementia or mental abnormality. They mentioned that the cortical hyperexcitability may attain rhyth-micity in posture, resulting in the clinical appearance of a tremor. Our case had tremor with familiarity, history of seizure; physiological findings fulfilled criteria of cortical reflex myoclonus; giant SEPs, enhancement of C-reflex, premyoclonus cortical discharge by jerk-locked averaging method [2-5], but no other neurological signs. From these characteristics our case was identified as a cortical tremor. Toro et al. [7], who studied cases of cortical myoclonus including cases without C-reflex, described that cortical tremor is common in patients with cortical myoclonus and that this phenomenon depends

on a central generator rather than on peripheral feedback loops, because transcranial magnetic stimulation but not peripheral nerve stimulation influenced tremor rhythm. In our case, peripheral nerve stimulation generated repetitive muscle activities, of which the interval was comparable to the frequency of the tremor. The first response was regarded to be C-reflex as long-loop reflex and the second or third responses should also be regarded as C-reflex. Tobimatsu et al. [6] also described repetitive C-reflexes in patients with sialidosis. These findings may be important on viewing the generating mechanism of cortical tremor that the presence of repetitive C-reflexes by a peripheral nerve stimulation as in our case may be closely related to a cortical tremor.

\section{References}

1 Ikeda A, Kakigi R, Funai N, Kuroda Y, Shibasaki H: Cortical tremor: A variant of cortical reflex myoclonus. Neurology 1990;40:1561-1565.

2 Kakigi R, Shibasaki H: Generator mechanisms of giant somatosensory evoked potentials in cortical reflex myoclonus. Brain 1987; 110:1359-1373.

Rothwell JC, Obesco JA, Marsden CD: Electrophysiology of somatosensory reflex myoclonus. Adv Neurol 1986;43:385-398.

Shibasaki H, Yamashita Y, Neshige R, Tobimatsu S, Fukui R: Pathogenesis of giant somatosensory evoked potentials in progressive myoclonic epilepsy. Brain 1985;108:225-240.

5 Shibasaki H, Yamashita Y, Tobimatsu S, Neshige R: Electroencephalo-graphic correlates of myoclonus. Adv Neurol 1986;43:357-372.

6 Tobimatsu S, Fukui R, Shibasaki H, Kato M, Kuroiwa Y: Electrophysiological studies of myoclonus in sialidosis type 2. Electroenceph Clin Neuro-physiol 1985;60:16-22.

7 Toro C, Pascual-Leone A, Deuschl G, Tate E, Pranzatelli MR, Hallet M: Cortical tremor. A common manifestation of cortical myoclonus. Neurology 1993;43:2346-2353. 